\title{
Aspiration Pneumonia Resulting from a Peanut
}

\author{
Shinichi Matsuzaki, ${ }^{1,2}$ Hitoshi Yamashita, ${ }^{1}$ Katsuaki Endou, ${ }^{1}$ \\ Takehiko Araki, ${ }^{1}$ Satoshi Ban, ${ }^{3}$ Katue Yoshida, ${ }^{3}$ \\ Tamotsu Ishizuka, ${ }^{2}$ Sakae Maruta ${ }^{1}$ and Masatomo Mori ${ }^{2}$
}

\begin{abstract}
A 65-year-old woman was admitted to our hospital after aspirating a peanut. Bronchoscopic examination revealed obstruction of the right basal bronchus. Use of clamps achieved incomplete removal. Hemoptysis appeared after a few days. Computed tomography (CT) of the lung revealed consolidation in the right lower segment. Methylprednisolone and antibiotics improved symptoms, but chest CT revealed persistent consolidation. Consolidation improved after bronchoalveolar lavage (BAL) of the right basal bronchus during bronchoscopic re-examination. BAL is useful in treating pneumonia resulting from an aspirated foreign body. (Kitakanto Med J $2009 ; 59: 161 \sim 163$ )
\end{abstract}

Key Words : aspiration pneumonia, peanut, adult, bronchoalveolar lavage

\section{Introduction}

Tracheo-bronchial aspiration of foreign bodies typically occurs in children, but can occur in adults under certain circumstances. Diagnosis is often difficult and requires a high index of clinical suspicion. We discuss this adult case with a brief review of the literature.

\section{Case Report}

A 65-year-old woman visited our hospital due to wheezing and dyspnea after aspirating a peanut during a meal that day. Symptoms remained unimproved despite administration of asthma treatment with methylprednisolone. The patient had never smoked and had no significant past medical history.

Chest radiography showed no signs of a foreign body, atelectasis or hyperexpansion. Flexible bronchoscopy revealed an immature white lesion, probably a peanut, lodged in the right basal bronchus (Fig. 1). Symptoms improved after bronchoscopic examination, although cleaning using clamps had been incomplete. Hemoptysis subsequently developed after a few days. Computed tomography (CT) of the lung revealed consolidation in the right lower segment (Fig. 2). Treatment was initiated with methylprednisolone, anti- biotics, tranexamic acid and carbazochrome sodium sulfonate. Wheeze, dyspnea and hemoptysis improved, but findings on chest CT did not. Bronchoscopic examination was again performed for diagnosis and treatment, revealing no bleeding or coagulation, but identifying mucous embolic material remaining in the right basal bronchus (Fig. 3). Residual flecks were removed using biopsy forceps, then bron-

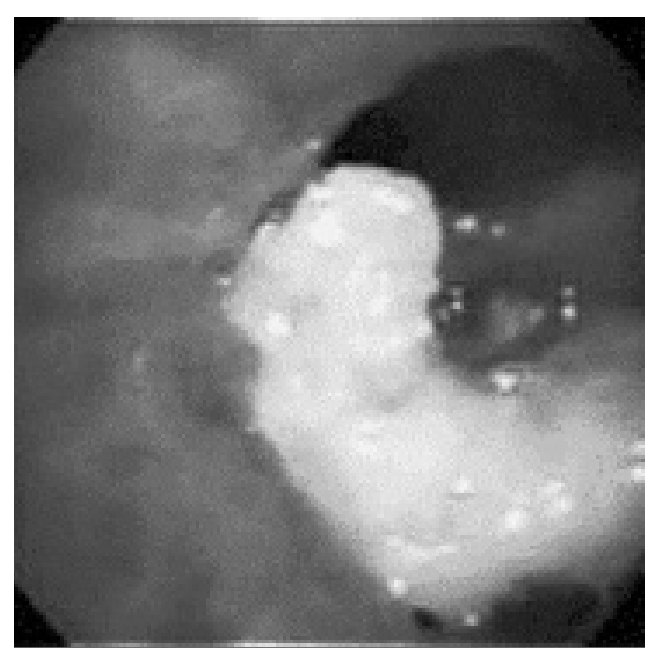

Fig. 1 Initial bronchoscopic findings. The right basal bronchus is obstructed by an immature white lesion, probably a peanut.

1 Department of Internal Medicine, Kiryu Kosei General Hospital, Kiryu, Japan 2 Department of Medicine and Molecular Science, Gunma University Graduate School of Medicine, Showa-machi, Maebashi, Gunma 371-8511, Japan 3 Department of Pathology, Kiryu Kosei General Hospital, Kiryu, Japan

Received : January 29, 2009

Address: SHINICHI MATSUZAKI Department of Medicine and Molecular Science, Gunma University Graduate School of Medicine, 3-39-15 Showa-machi, Maebashi, Gunma 371-8511, Japan 


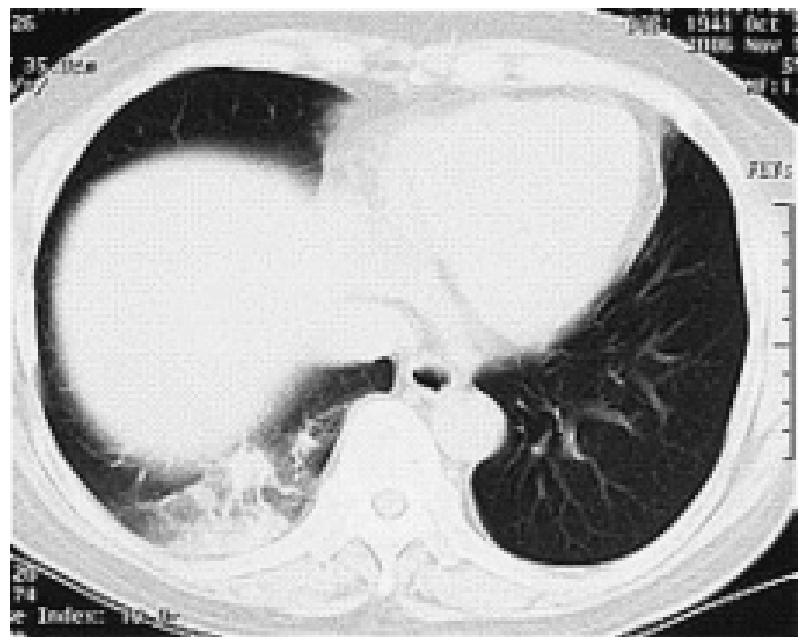

Fig. 2 Chest CT shows consolidation and peripheral groundglass opacity in the right lower lung field.

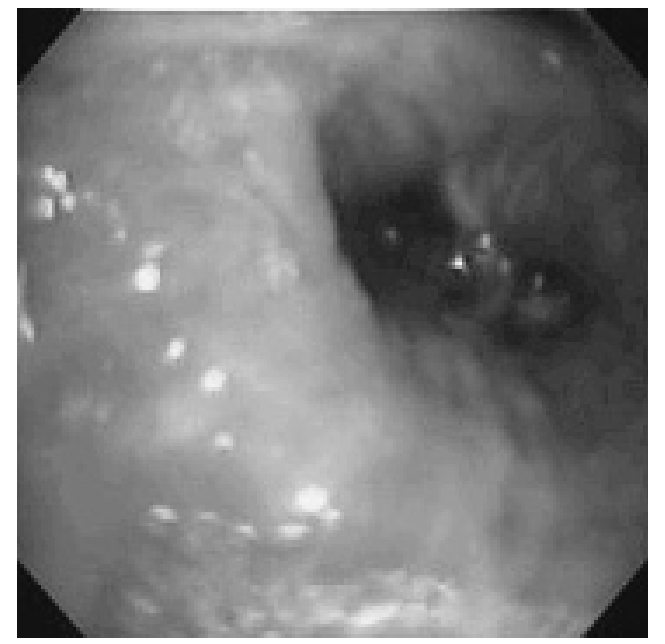

Fig. 3 Bronchoscopic findings after steroid and antibiotic treatment. The basal bronchus is obstructed by granulation.

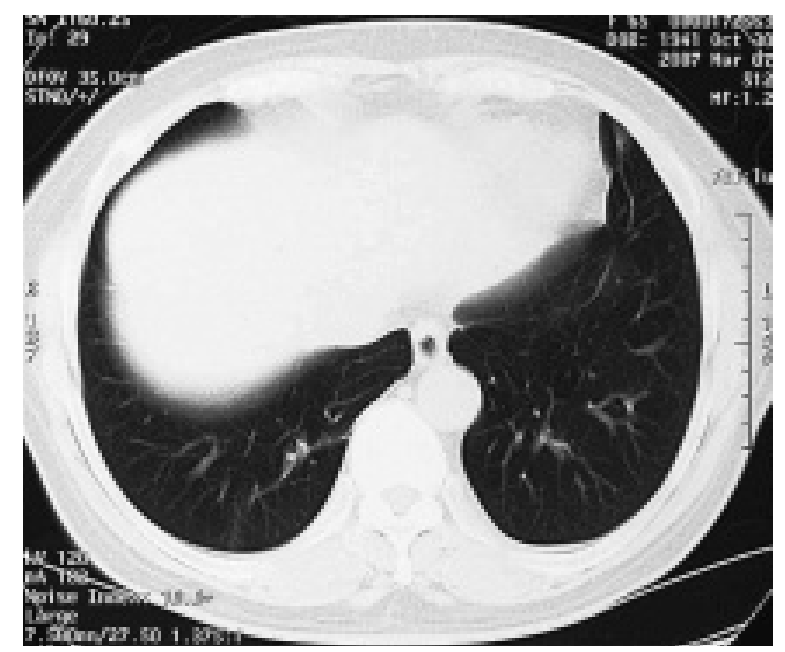

Fig. 4 Chest CT 2 weeks after BAL shows only linear shading in the right lower lung field.

choalveolar lavage (BAL) was performed with approximately $100 \mathrm{ml}$ of saline. After treatment, chest CT revealed improvements in consolidation and clinical recovery was uneventful without recurrence (Fig. 4).

\section{Discussion}

Aspirated foreign body ( $\mathrm{AFB}$ ) is more common in children than in adults. Most AFBs in children have been noted to be organic, with beans accounting for $60-65 \%$, and peanuts making up a proportion as high as $31-46 \%{ }^{1} \quad$ However, aspiration of a peanut is rare in adults. Mean time to diagnosis is significantly shorter in children than in adults, and AFBs can remain undetected in adults for several months.

The diagnostic clinical triad described for pediatric AFB is wheezing, cough and decreased breath sounds. Adults can present with non-specific signs and symptoms such as chronic cough, hemoptysis and dyspnea, and stridor, cyanosis, tachypnea, vomiting and hoarseness have also been described. The most frequently reported symptom is penetration syndrome, defined as a sudden onset of choking and intractable cough with or without vomiting, found in $49 \%$ of patients. $^{2}$ In this case, preceding treatment for asthma attack was performed because adult AFB was not recognized.

Air trapping is the predominant radiographic finding in child, whereas atelectasis is more common in adults. Sensitivity of plain radiography is reportedly $62 \% .^{3} \mathrm{CT}$ and magnetic resonance imaging appear useful as the initial imaging modality for suspected AFB.

Definitive treatment of AFB involves removal of the foreign body. Rigid bronchoscopy is used as the first-line therapy due to the possibility of residual fragments with flexible bronchoscopy and success depends on operator skill.

In this case, we initially used a flexible bronchoscope because the patient did not agree to a procedure using a rigid bronchoscope. In general, the vegetable foreign body reaction for a peanut is stronger than a non-vegetative one. ${ }^{4}$ Abruption was difficult due to strong inflammatory changes. Peanut aspiration was suspected as the cause of obstructive pneumonia. Systemic steroid treatment was considered unlikely to decrease bronchial inflammation. After BAL, chest CT revealed a marked improvement in consolidation. BAL involves the lavage of bronchoalveolar secretions from the lower respiratory tract and is an important procedure that can be performed during bronchoscopy. This technique is a useful and safe method for sampling cellular and biochemical components from the lung. BAL also plays an important role in the therapeutic management of AFB. Some reports have described bronchoscopic intralesional injection of triamcinolone acetonide as useful for obstruction of the bronchus by granulation tissue caused by AFB. ${ }^{5}$ 
In conclusion, we reported a case in which BAL improved pneumonia caused by aspiration of a peanut by a healthy adult. Careful attention should be paid to the signs and symptoms of AFB in adults. BAL appears useful in treating obstructive pneumonia due to AFB. Further studies are needed to determine effective treatments for aspiration pneumonia due to AFB.

\section{Reference}

1. Samuel CLL, Kaleelullah SF : Peanut aspiration in an adult. Prim Care Respir J. 2004 ; 13 : 169-170.

2. Barharloo F, Veyckemans F, Francis C, Biettlot MP, Rodenstein DO. Tracheobronchial foreign bodies: Presentation and management in children and adults. Chest. $1999 ; 115$ : 1357-1362.

3. Mu LC, Sun DQ, He P. Radiological diagnoses of aspirated foreign bodies in children : review of 343 cases. J Laryngol Otol. $1990 ; 104$ : 778-782.

4. Takao H. Supplemental experimental findings on foreign body in the bronchus. J Jpn Bronchoesophagol Soc. 1973; 24 : 30-39.

5. Niwa $T$, Nakamura A, Kato $T$, Kutsuna $T$, Tonegawa $K$, Kato $\mathrm{K}$, et al. Bronchoscopic intralesional injection of triamcinolone acetonide treated against bronchial obstruction caused by peanut aspiration. Respir Med. 2005; 99 : 645-647. 